\title{
Terahertz Faraday rotation in a magnetic liquid: High magneto-optical figure of merit and broadband operation in a ferrofluid
}

\author{
Mostafa Shalaby, ${ }^{1, \text { a) }}$ Marco Peccianti, ${ }^{2, b)}$ Yavuz Ozturk, ${ }^{1,3}$ Matteo Clerici, ${ }^{1}$ \\ Ibraheem Al-Naib, ${ }^{1}$ Luca Razzari, ${ }^{4}$ Tsuneyuki Ozaki, ${ }^{1}$ Anna Mazhorova, ${ }^{5}$ \\ Maksim Skorobogatiy, ${ }^{5}$ and Roberto Morandotti ${ }^{1}$ \\ ${ }^{1}$ INRS-EMT, Varennes, Québec J3X 1S2, Canada \\ ${ }^{2}$ ISC-CNR, Via dei Taurini 19, 00185 Rome, Italy \\ ${ }^{3}$ Ege University, 35100 Izmir, Turkey \\ ${ }^{4}$ Italian Institute of Technology, 16163 Genova, Italy \\ ${ }^{5}$ Ecole Polytechnique de Montreal, Genie Physique, Québec, H3C 3A7, Canada
}

(Received 13 April 2012; accepted 26 May 2012; published online 13 June 2012)

\begin{abstract}
We report on the demonstration of a high figure of merit (FOM) Faraday rotation in a liquid in the terahertz $(\mathrm{THz})$ regime. Using a ferrofluid, a high broadband rotation $(11 \mathrm{mrad} / \mathrm{mm})$ is experimentally demonstrated in the frequency range of $0.2-0.9 \mathrm{THz}$ at room temperature. Given the low absorption of the liquid, a high magneto-optical figure of merit $(5-16 \mathrm{rad} . \mathrm{cm} / \mathrm{T})$ is obtained. (C) 2012 American Institute of Physics. [http://dx.doi.org/10.1063/1.4729132]
\end{abstract}

Ferrofluids, colloidal suspensions of ferrimagnetic nanoparticles in a carrier liquid, underwent an extensive investigation, since their first development in early 1960s. ${ }^{1}$ While based on a ferromagnetic material, they behave as paramagnets and show net magnetization only under the application of external magnetic fields. ${ }^{2}$ The flexibility of the liquid form and the low magnetic field requirements to observe the desired effects give them a significant advantage over their solid counterparts for a wide range of applications. For example, they can be used as an infiltrated core of dielectric waveguiding structures. In a normally isotropic ferrofluid, an external magnetic field parallel or orthogonal to the polarization plane of a propagating electromagnetic wave can induce linear (Voigt) or circular (Faraday) birefringence, respectively. Faraday rotation (FR) in ferrofluids, for example, has been demonstrated in the visible, ${ }^{3,4}$ near-infrared, ${ }^{5,6}$ and mid-infrared ${ }^{7}$ regimes. Here, we investigate FR at the terahertz $(\mathrm{THz})$ frequencies. Specifically, we demonstrate that in ferrofluids, $\mathrm{THz}$ waves undergo strong FR with remarkably low net absorption at room temperature, paving the way to $\mathrm{THz}$ non-reciprocal devices.

FR in the $\mathrm{THz}$ regime has been demonstrated in solids at low temperatures. ${ }^{8}$ At room temperature, Nakajima et al. showed narrow band FR induced by a sharp ferromagnetic resonance. ${ }^{9}$ Broadband FR has been demonstrated in doped silicon $^{10}$ and HgTe thin films. ${ }^{11}$ In the latter, a high magneto-optical figure of merit (FOM), i.e., the FR normalized by the absorption coefficient, is reported with a significant polarization rotation below $0.5 \mathrm{THz}$. However, such high FR and FOM in HgTe decrease dramatically as the thickness increases.

In this work, we report a high THz FOM in ferrofluids. In comparison with the aforementioned studies, the presented results are not critically dependent on the thickness and the medium exhibits almost constant FR within 0.2-0.9 $\mathrm{THz}$, suitable for $\mathrm{THz}$ radiation from broadband ${ }^{12}$ and tuna-

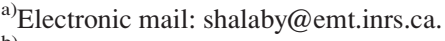

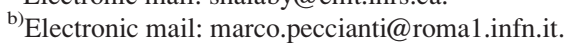

ble sources. ${ }^{13}$ These properties are of potential importance for future FR devices at $\mathrm{THz}$ frequencies. The nanoparticles in ferrofluids are usually coated with surfactants to prevent agglomeration. ${ }^{14,15}$ In this work, we use a commercial ferrofluid, EFH1 from Ferrotec (USA), consisting of $10 \mathrm{~nm}$-sized nanoparticles of $\mathrm{Fe}_{3} \mathrm{O}_{4}$ dispersed in a hydrocarbon carrier fluid. The particle concentration is $7.3 \%$ in weight and the fluid is confined in a cuvette $10 \mathrm{~mm}$-thick along the $\mathrm{THz}$ propagation direction. When no external magnetic field is applied, the particles are randomly distributed with zero coercivity and remanence and the fluid can be regarded as an isotropic material. An external magnetic field can trigger the coagulation of the particles that form chain-like clusters oriented in the field direction ${ }^{16,17}$ as illustrated in Fig. 1(a). The fluid exhibits a strong ferromagnetic polarizability along the magnetic field direction; hence electromagnetic fields propagating along that direction experience FR.

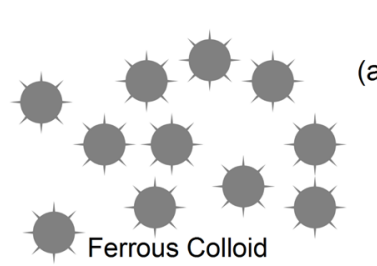

(b)

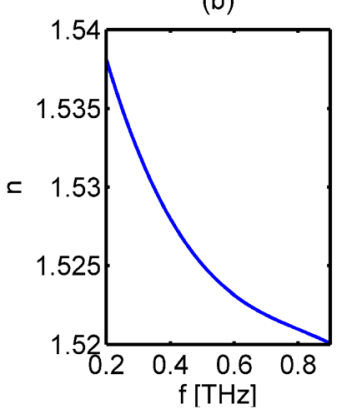

(a)

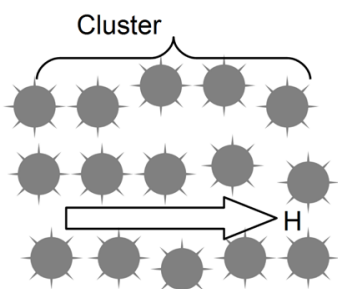

(c)

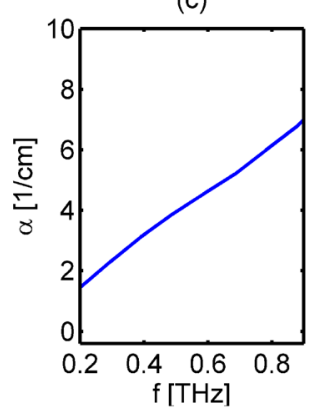

FIG. 1. (a) Magnetic nanoparticles alignment and chain formation in the direction of the applied magnetic field. (b) Refractive index and (c) absorption coefficient for EFH1 in the frequency range of $0.2-0.9 \mathrm{THz}$. 
The experimental setup consists of a conventional $\mathrm{THz}$ time domain spectroscopy system (THz-TDS). ${ }^{18}$ The system is powered by a $1 \mathrm{kHz}$ regenerative source (Spectra-Physics Spitfire) providing $1.1 \mathrm{~mJ}, 130 \mathrm{fs}$-wide pulses centered at $800 \mathrm{~nm}$. THz pulses are generated by means of optical rectification in a ZnTe crystal and detected using standard electro-optic (EO) sampling. The frequency-dependent dielectric function of the medium in the $0.2-0.9 \mathrm{THz}$ frequency range and in absence of an external magnetic field has been characterized by TDS and is shown in Fig. 1(b) (refractive index) and Fig. 1(c) (absorption coefficient). The absorption coefficient is as low as $1.8 \mathrm{~cm}^{-1}$ at $0.2 \mathrm{THz}$ and monotonically increases with frequency. This absorption is a collective effect of both the nanoparticles and carrier fluid. FR characterization has been performed using a standard cross ellipsometry polarization probing setup, ${ }^{10}$ consisting of a set of three wire-grid polarizers $\left(\mathrm{WGP}_{1} ; \mathrm{WGP}_{2} ; \mathrm{WGP}_{3}\right)$ placed in the THz optical path as depicted in Fig. 2.

We assumed that the electric and magnetic fields are linearly polarized along the $\mathrm{y}$ and $\mathrm{x}$ directions as defined by $\mathrm{WGP}_{1}$ and $\mathrm{WGP}_{3}$ (i.e., when the polarizers grid is along $\mathrm{x}-$ axis the transmission is maximum). The output polarization is probed by rotating the polarization axis of $\mathrm{WGP}_{2}$ along the $x$-axis by $45^{\circ}$ and $-45^{\circ}$. An external magnetic field, collinear with the $\mathrm{THz}$ propagation direction, is applied using a commercial electromagnet, GMW-3470. Removing the magnet poles concentrators ensured uniform magnetic field along the sample volume.

The calculation of the FR requires the estimation of its radiation eigenmodes in the magnetic medium, i.e., the right circularly polarized (RCP) and left circularly polarized (LCP) waves. RCP and LCP can be calculated from the measurement of the linear components by simply considering the vectorial identity

$$
\left(\begin{array}{l}
E_{1}(f) \\
E_{\mathrm{r}}(f)
\end{array}\right)=\frac{1}{2}\left(\begin{array}{cc}
-1+i & 1+i \\
1+i & -1+i
\end{array}\right)\left(\begin{array}{c}
E_{+45}(f) \\
E_{-45}(f)
\end{array}\right)
$$

where $\mathrm{E}_{\mathrm{r}}$ and $\mathrm{E}_{1}$ are the RCP and LCP components. $\mathrm{E}_{+45}$ and $\mathrm{E}_{-45}$ are the detected $\mathrm{THz}$ components when $\mathrm{WGP}_{2}$ is rotated by $45^{\circ}$ and $-45^{\circ}$, respectively. The FR angle $\theta$ and ellipticity $\psi$ (Ref. 19) are

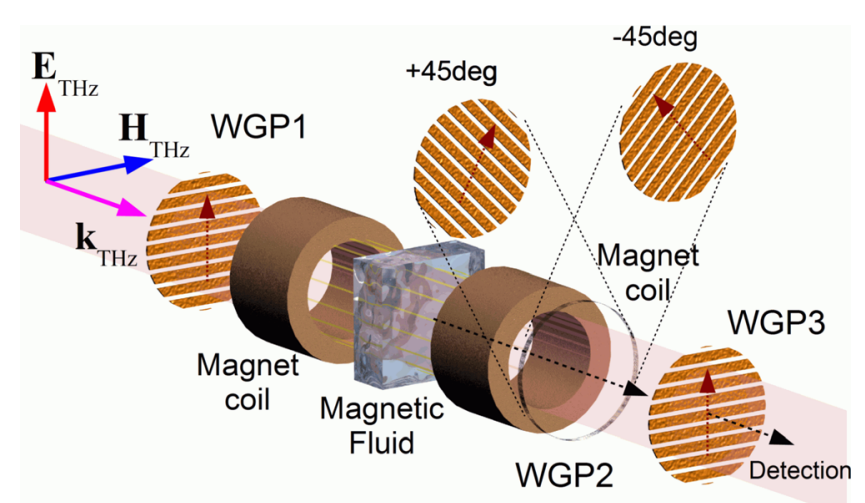

FIG. 2. Schematic diagram of a cross ellipsometry setup employed to measure FR and ellipticity. $\mathrm{WGP}_{1}$ and $\mathrm{WGP}_{3}$ are set to $0^{\circ}$. $\mathrm{WGP}_{2}$ is rotated by $45^{\circ}$ and $-45^{\circ}$ from the $0^{\circ}$ position.

$$
\theta(f)=\frac{\arg \left[E_{\mathrm{r}}(f)\right]-\arg \left[E_{\mathrm{l}}(f)\right]}{2}
$$

and

$$
\psi(f)=\frac{\left|E_{\mathrm{l}}(f)\right|-\left|E_{\mathrm{r}}(f)\right|}{\left|E_{\mathrm{l}}(f)\right|+\left|E_{\mathrm{r}}(f)\right|} .
$$

As shown in Fig. 1(a), the THz absorption increases with frequency. Due to the large sample thickness, the signal to noise ratio $(\mathrm{S} / \mathrm{N})$ degrades significantly above $1 \mathrm{THz}$. Hence we limited our characterization to the $0.2-0.9 \mathrm{THz}$ range. The external magnetic field has been varied from -30 to $30 \mathrm{mT}$. While the sample always induced a negligible ellipticity under the applied magnetic field, strong FR is observed as shown in Fig. 3(a). The field transmitted through the medium exhibits a high rotation that depends weakly on the $\mathrm{THz}$ field frequency, i.e., is featured by a broadband operation. A FR angle as large as $110 \mathrm{mrad}$ is measured at $30 \mathrm{mT}$, a remarkably small magnetic field.

Under an applied magnetic field, the magnetic dipole moments start to align with the field. This is counteracted by the thermal energy that tends to randomize the orientation of the dipole moments. The average number of magnetic moments aligned to the external field can be statistically estimated. The net magnetization (M) is theoretically found ${ }^{20}$ to have a Langevian behavior where $\mathrm{M} / \mathrm{M}_{s}=\operatorname{coth}(\mathrm{kH})-1 /(\mathrm{kH})$. Here, $\mathrm{k}$ is a temperature-dependent parameter. $\mathrm{M}_{s}$ is the saturation magnetization, which is nominally $40 \mathrm{mT}$ for $\mathrm{EFH} 1$

(a)

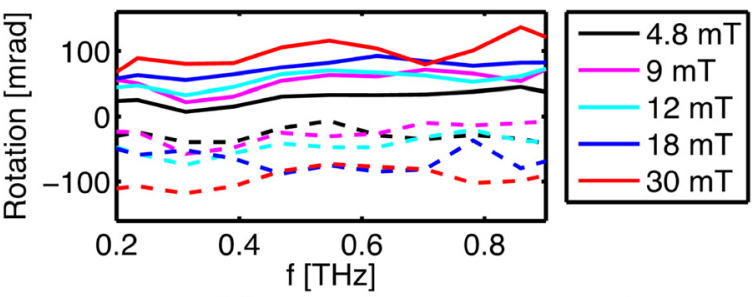

(b)

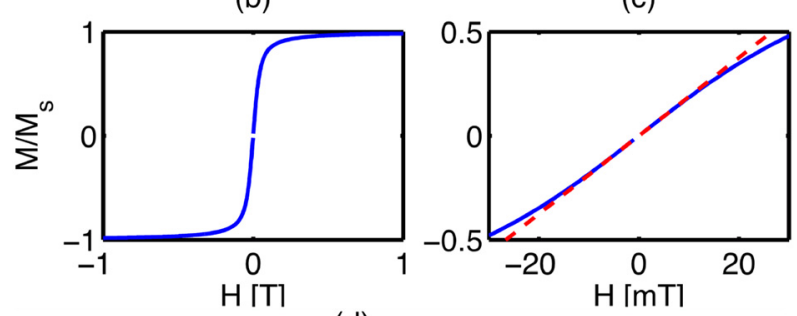

(d)

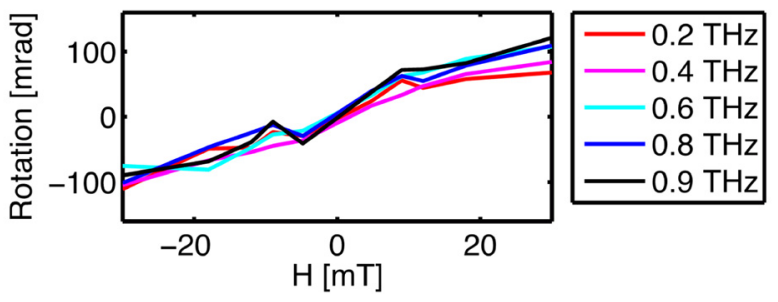

FIG. 3. (a) FR measurements in the spectral range $0.2-0.9 \mathrm{THz}$ for different levels of applied magnetic field $(\mathrm{H})$. The dotted curves show the corresponding rotation when the external magnetic field direction is reversed. The Langevian behavior of magnetization is shown in (b) and zoomed in (c) along with a kH/3 linear approximation shown by red dashed lines. (d) FR measurements plotted against $\mathrm{H}$ for certain values of frequency. 
(a) Verdet Constant (V)

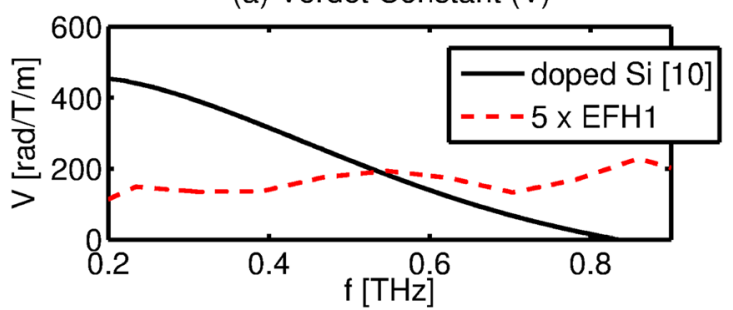

(b) Figure of Merit (FOM)

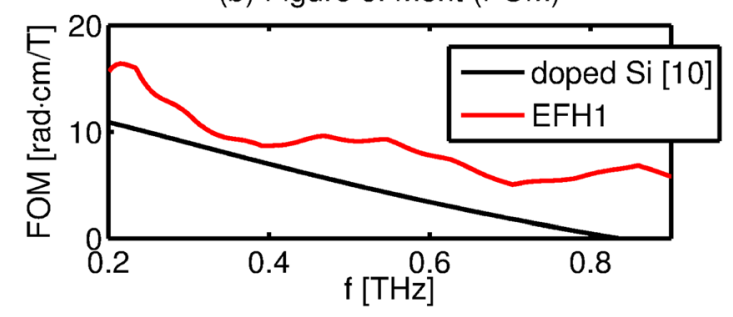

FIG. 4. Comparison between the presented EFH1 and a literature case of doped $\mathrm{Si}^{10}$ (a) Verdet constant and (b) FOM.

under an applied magnetic field of $1 \mathrm{~T}^{20}$ At the field levels used in our experiments $(<30 \mathrm{mT})$, the net magnetization is in the nearly linear regime and $\mathrm{M} / \mathrm{M}_{s}$ can be approximated to $\mathrm{kH} / 3 .^{20}$ This is depicted in Figs. 3(b) and 3(c). The induced magnetization and thus FR are expected to have a linear dependence on the applied magnetic field. This theoretical prediction is confirmed in Fig. 3(d) where the FR shows an almost-linear dependence on the applied field for different, arbitrarily selected frequency lines, i.e., 0.2, 0.4, 0.6, 0.8, and 0.9 THz. According to Fig. 3(c), an applied magnetic field of $30 \mathrm{mT}$ corresponds to $0.5 \mathrm{M}_{s}$ for EFH1 with a particle size of $10 \mathrm{~nm}$. A high rotation at low magnetic fields and a quick onset of the nonlinear and saturation regimes give to this magnetic fluid a stark advantage in low magnetic field applications at $\mathrm{THz}$ frequencies. For example, such low fields can be obtained from magnetic heads in computer memories. When this advantage adds up to the liquid nature of our material, the possible range of applications widens.

Accumulating a large rotation over long propagation lengths requires high transparency of the material in the desired frequency band. The FOM enables the comparison between magneto-optic materials in terms of the largest achievable rotation for a given absorption. EFH1 offers high transparency in the considered $\mathrm{THz}$ band. A number of solutions have been proposed for THz-FR at room temperature. ${ }^{9-11} \epsilon-\mathrm{Fe}_{2} \mathrm{O}_{3}$ (Ref. 9) shows a sharp narrowband resonance-induced FR while the occurrence of FR in $\mathrm{HgTe}$ (Ref. 11) depends strongly on the film thickness. Furthermore, large FRs have been only realized for ultra-thin films of HgTe. Figure 4 compares EFH1 with the only similar case of THz-FR, which was observed in doped silicon. ${ }^{10}$ Doped silicon exhibits a much stronger FR frequency dependence. Although EFH1 exhibits a lower Verdet constant (rotation angle normalized by sample thickness and magnetic field), its higher FOM leads to higher rotations for a given Faraday rotator loss.

In conclusion, we demonstrated FR in a liquid in the $\mathrm{THz}$ regime. Using a commercial ferrofluid, we obtained a broadband rotation $(11 \mathrm{mrad} / \mathrm{mm})$ in the $0.2-0.9 \mathrm{THz}$ range at room temperature. The liquid considered shows high transparency in the $\mathrm{THz}$ regime that in turn leads to a high FOM. Considering the flexibility of the liquid form and the low-magnetic field requirements, we believe that our results will lead to a wide range of applications in the field of $\mathrm{THz}$ metamaterials and magneto-optics.

We would like to thank Dr. Osamu Morikawa (Japan Coast Guard Academy) for providing us with raw data ${ }^{10}$ of his relevant work for comparison. This work is supported by the FQRNT (Le Fonds Qubcois de la Recherche sur la Nature et les Technologies) and the NSERC (the Natural Sciences and Engineering Research Council of Canada). M.S. and M.C. wish to acknowledge ongoing FQRNT MELS scholarships.

${ }^{1}$ S. S. Papell, U.S. Patent 3,215,572 (1965).

${ }^{2}$ C. P. Bean, R. W. DeBlois, and L. B. Nesbitt, J. Appl. Phys. 30, 1976 (1959).

${ }^{3}$ N. A. Yusuf, A. A. Rousan, and H. M. El-Ghanem, J. Appl. Phys. 64, 2781 (1988).

${ }^{4}$ Z. Di, X. Chen, S. Pu, X. Hu, and Y. Xia, Appl. Phys. Lett. 89, 211106 (2006).

${ }^{5}$ Y. T. Pan, C. W. Du, X. D. Liu, Z. G. Li, and R. Birngruber, J. Appl. Phys. 73, 6142 (1993).

${ }^{6}$ X. Fang, Y. Xuan, and Q. Li, Nanoscale Res. Lett. 6, 237 (2011).

${ }^{7}$ M. Maiorov, J. Magn. Magn. Mater. 252, 111 (2002).

${ }^{8}$ J. Van Slageren, S. Vongtragool, A. Mukhin, B. Gorshunov, and M. Dressel, Phys. Rev. B 72, 020401 (2005).

${ }^{9}$ M. Nakajima, A. Namai, S. Ohkoshi, and T. Suemoto, Opt. Express 18, 18260 (2010).

${ }^{10}$ O. Morikawa, A. Quema, S. Nashima, H. Sumikura, T. Nagashima, and M. Hangyo, J. Appl. Phys. 100, 033105 (2006).

${ }^{11}$ A. M. Shuvaev, G. V. Astakhov, A. Pimenov, C. Brüne, H. Buhmann, and L. W. Molenkamp, Phys. Rev. Lett. 106, 107404 (2011).

${ }^{12}$ Y. Shen, T. Watanabe, D. A. Arena, C.-C. Kao, J. B. Murphy, T. Y. Tsang, X. J. Wang, and G. L. Carr, Phys. Rev. Lett. 99, 043901 (2007).

${ }^{13}$ W. Shi, Y. Ding, N. Fernelius, and K. Vodopyanov, Opt. Lett. 27, 1454 (2002).

${ }^{14}$ R. E. Rosensweig, Nature (London) 210, 613 (1966).

${ }^{15}$ M. Shliomis, Sov. Phys. JETP 34, 1291 (1972).

${ }^{16}$ C. Rablau, P. Vaishnava, C. Sudakar, R. Tackett, G. Lawes, and R. Naik, Phys. Rev. E 78, 051502 (2008).

${ }^{17}$ A. Rousan, H. El-Ghanem, and N. Yusuf, IEEE Trans. Magn. 25, 3121 (1989).

${ }^{18}$ M. van Exter and D. Grischkowsky, Appl. Phys. Lett. 56, 1694 (1990).

${ }^{19}$ E. D. Palik and J. K. Furdyna, Rep. Prog. Phys. 33, 1193 (1970).

${ }^{20}$ T. A. Franklin, "Ferrofluid flow phenomena," Master's thesis (Massachusetts Institute of Technology, 2003). 